Research Article

\title{
Use of a Secondary Current Sensor in Plasma during Electron-Beam Welding with Focus Scanning for Process Control
}

\author{
Dmitriy Trushnikov, ${ }^{1}$ Elena Krotova, ${ }^{1}$ and Elena Koleva ${ }^{2}$ \\ ${ }^{1}$ Perm National Research Polytechnic University, 29 Komsomolsky Av., Perm 614990, Russia \\ ${ }^{2}$ Institute of Electronics, Bulgarian Academy of Sciences, 72 Tzarigradsko Shose, 1784 Sofia, Bulgaria \\ Correspondence should be addressed to Dmitriy Trushnikov; trdimitr@yandex.ru
}

Received 14 August 2016; Accepted 12 October 2016

Academic Editor: Stephane Evoy

Copyright (C) 2016 Dmitriy Trushnikov et al. This is an open access article distributed under the Creative Commons Attribution License, which permits unrestricted use, distribution, and reproduction in any medium, provided the original work is properly cited.

\begin{abstract}
We consider questions of building a closed-loop focus control system for electron-beam welding. As a feedback signal, we use the secondary current in the plasma that forms above the welding zone. This article presents a model of a secondary current sensor in plasma during electron-beam welding with focus scanning. A comparison of modeled results with experimental data confirms the adequacy of the model. We show that the best results for focus control are obtained when using phase relationships rather than amplitude relationships. We outline the principles for building an EBW focus control system based on parameters of the secondary current in plasma. We simulate the work of a control system's circuits and demonstrate the stability of the synthesized system. We have conducted pilot tests on an experimental prototype.
\end{abstract}

\section{Introduction}

Electron-beam welding (EBW) is widely used in such hightech fields of industry as power engineering and aerospace. This is due to EBW's ability to produce deep melting, where the ratio of the weld depth to weld width is large with minimal metal deformation and small heat-affected zone width.

A large number of works [1-5] have been devoted to studying the mechanisms and laws of melting, without knowledge of which it is impossible to use electron beams to process materials. Despite the successes achieved in studying this process, quite a few problems remain unsolved in EBW theory and practice. Forming a welded joint during EBW is a complex process resulting from the combined influence of many factors caused by the effect of a powerful concentrated energy source (electron beam) on the materials being welded; see [5-10]. It is typically difficult to reproduce high-quality welded joints, despite having stabilized all of the electric characteristics of the devices feeding the electron-beam apparatuses. In most cases, EBW is applied when producing especially critical joints in complex and expensive products (gas-turbine engine parts for airplanes, spacecrafts, helicopter gears, etc.), and defects caused by an incorrect choice of electron-beam focus could have catastrophic consequences.

Adaptive control makes it possible to ensure consistent quality during welding and related processes [11-16]. Its measures can reduce the size of allowances in subsequent mechanical processing, leading to a reduction in the cost of production and improving the energy-efficiency of the process. In addition to solving the problems mentioned above, using this operational control and monitoring makes it possible to significantly reduce expenses on the optimization of processes, which also makes it possible to reduce the cost of production overall.

The connection between X-ray radiation and the technical parameters of electron-beam welding has been quite thoroughly studied [17, 18]. Valuable results have been obtained on controlling the aim of the electron beam as well as on automatically controlling the penetration depth in welding of aluminum alloys. Significantly less attention has been given to stabilizing the focusing conditions directly during welding. 


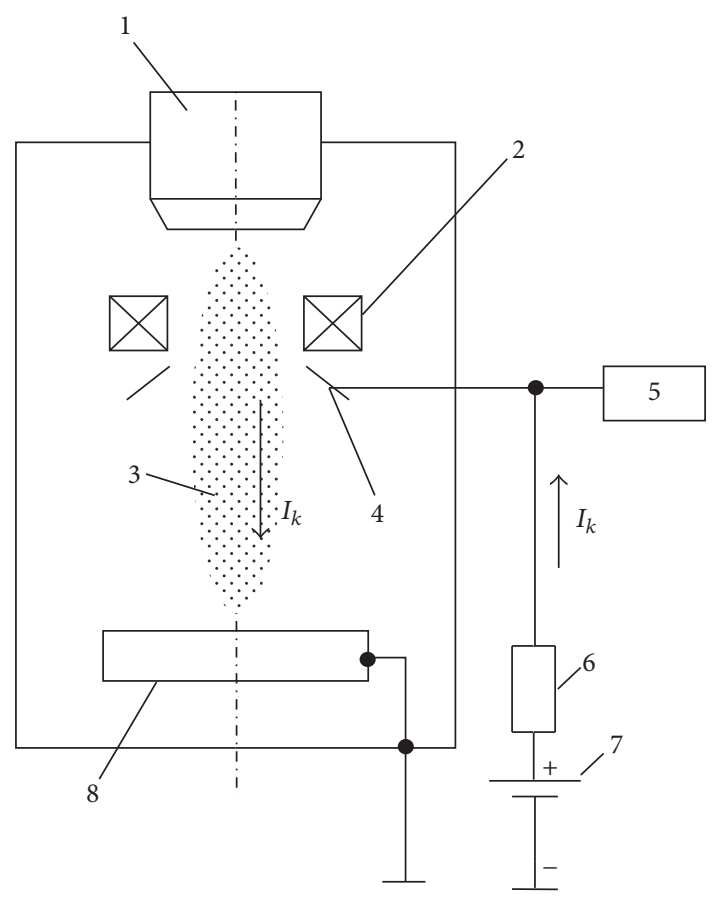

FIGURE 1: Scheme of secondary electron current registration in the plasma formed above the zone of EBW: 1, electron gun; 2, focusing lens; 3, plasma generated above the welding zone; 4, collector; 5, current registration and memory of the measured data; 6 , load resistor; 7 , voltage source; 8 , welded sample.

There are well-known preliminary setup methods that take advantage of an analysis of the beam density distribution using special sensors and computed tomography algorithms [19-23], but using these methods directly in the welding process is problematic and requires periodical interruption of the process, which has a negative effect on the quality of the welded joints.

One of the specific processes caused by the impact of the intensive electron beam on the metal during EBW is the formation of plasma in the operational area $[6,7]$. The parameters of the plasma are closely connected with the electron-beam thermal effect on the metal being welded. In $[8,9]$, the plasma current parameters are suggested for electron-beam focusing control. The scheme for the registration of the collected plasma current is shown in Figure 1 [12]. Highlighted is a standard electron-beam welding machine with a thermionic electron gun (1), with a focusing coil (2) at the end, opposite a welded specimen (8), all situated in a vacuum chamber. The electron beam, generated from the electron gun, is directed and focused on the specimen surface. The vapour/plasma streams are collimated by keyhole, which directs their expansion upwards. In the case of the positive collector electrode immersed in the plasma plume, the discharge glows between the two electrodes, the grounded specimen (cathode) and the ring collector of the plasma electrons (anode). The collector (4) is situated below the end of the electron gun in the grounded vacuum metallic chamber. In the outer collector circuit trough, a load resistor
(6) of $50 \Omega$ is connected to a positive voltage of $50 \mathrm{~V}$. The measuring and memory block is signed as (5).

All known methods of electron-beam focusing control use extreme correlations between the secondary emissions and the focusing lens current. These correlations are characterized by the dead zones and two values of the focusing lens current that ensure similar signal parameters. When building automatic focus control systems there is a natural desire to use high-frequency scanning of the focal spot [2427]. However, until now the formation of a secondary current waveform in plasma in this case has not been investigated. Until recently, the literature has lacked information about models that describe the formation of a secondary waveform during EBW with scanning of the electron beam's focus. In building controllers, well-known works use relationships obtained during research under static conditions. In this case, it is unknown whether the extreme behavior of the electric current in the plasma as a function of the focus current is caused by dependence on the energy density of the electron beam or it is linked to the changing geometry of the penetration channel. As a result, there are no recommendations regarding limitations applied to the choice of frequency for scanning the focus of the electron beam.

Reference [12] demonstrates that a coherent accumulation method makes it possible to develop new EBW control methods. The advisability of moving to phase parameters is shown. When processing the waveform of the secondary current in plasma using a synchronous accumulation method the secondary waveform is seen to lag relative to the current of the deflection coils. The lag value characterizes the inertia of the heating process in the interaction zone. Maintaining a constant lag makes it possible to stabilize the intensity of heating the metal in the penetration channel, regardless of external parameters (initial temperature, welding speed, oscillation parameters, etc.). However, the work lacks any reasoning for the occurrence of this lag or the nature of the change in lag. Additionally, $[12,24]$ hardly illuminate the questions of organizing a control system that uses the waveform from the described sensor.

\section{Results and Discussion}

2.1. Model of the Formation of a Secondary Waveform during $E B W$. Studying the secondary current using an electron collector, which is positioned above the welding puddle and used to sample the current from the plasma, has shown that in addition to the beam current the size of this current is determined by the shape and dimensions of the penetration channel, conditions of the flow of current in the plasma, the position of the beam's area of interaction with the metal, the position of the beam's focus in the penetration channel, and so forth $[28,29]$. Therefore, the secondary waveform from a non-self-sustained discharge of current in plasma may be expressed as follows [30]:

$$
I_{c}=F(Y) \cdot I_{b},
$$

where $Y$ is the vector of parameters $\left(y_{1}, y_{2}, \ldots, y_{n}, t\right)$ and $F(Y)$ is a function of this vector that reflects the stochastic nature of the process of electron moving outward through 
the channel, which contains all of the factors influencing the size of the detected current, except the current of the electron beam.

In formula (1), the function $F(Y)$ can be broken down into the sum of three parts that represent components dependent on and independent of the focus current and a part dependent on the rate of change of the focus current:

$$
F(Y)=F_{1}\left(Y_{1}\right)+F_{2}\left(I_{f}\right)+F_{3}\left(\frac{\partial I_{f}}{\partial t}\right)
$$

where $Y_{1}$ is an $n$-dimensional vector of parameters $\left(y_{1}, y_{2}\right.$, $\left.\ldots, y_{n}, t\right)$, which contains all of the factors influencing the size of the detected current, except the current of the electron beam, focus current, and rate of change of the focus current. We take $F_{3}\left(\partial I_{f} / \partial t\right)$ to be the proportional rate of change from the focus current. Furthermore, let $F_{3}\left(\partial I_{f} / \partial t\right)$ be proportional to the magnitude of the secondary current. In particular, it approaches zero when $F_{2}\left(I_{f}\right)$ approaches zero.

We assume that given high-frequency focus scanning (scanning focus $\omega>300 \mathrm{~Hz}$ ) the geometry of the penetration channel is not able to change significantly during the scanning period. Then the function $F_{2}\left(I_{f}\right)$ depends only on the focus current's influence on the flux density of the energy added by the electron beam.

We approximate this relationship, which would be observed given if an electron beam hit a flat specimen in the conditions present above the plasma's interaction zone, as a Gaussian function:

$$
F_{2}\left(I_{f}\right)=\frac{K}{\sigma \sqrt{2 \pi}} \exp \left[-\frac{\left(I_{f}-I_{f \mathrm{ext}}\right)^{2}}{2 \sigma^{2}}\right],
$$

where $K$ is a certain proportionality coefficient, $\sigma$ is a parameter that characterizes the shape of the function, $I_{f}$ is the focus current, and $I_{f \mathrm{ext}}$ is the value of the sharp focus current that maximizes the flux density of the energy added by the electron beam.

To achieve focus scanning, we introduce a harmonic oscillation of the focus current according to

$$
I_{f}=\left\langle I_{f}\right\rangle+I_{\mathrm{fm}} \sin (\omega \cdot t),
$$

where $I_{\mathrm{fm}}$ is the amplitude of the focus current modulation, $\left\langle I_{f}\right\rangle$ is the average value of the focus current, $\omega$ is the scanning frequency, and $t$ is the time.

Formula (3) becomes

$$
\begin{aligned}
F_{2}\left(I_{f}\right) & =\frac{K}{\sigma \sqrt{2 \pi}} \exp \left[-\frac{\left(\left\langle I_{f}\right\rangle+I_{\mathrm{fm}} \sin \alpha-I_{f \mathrm{ext}}\right)^{2}}{2 \sigma^{2}}\right] \\
& =\frac{K}{\sigma \sqrt{2 \pi}} \exp \left[-\frac{\left(\Delta I_{f}+I_{\mathrm{fm}} \sin \alpha\right)^{2}}{2 \sigma^{2}}\right],
\end{aligned}
$$

where $\alpha=\omega t, \Delta I_{f}=\left\langle I_{f}\right\rangle-I_{f \text { ext }}$ is the focus state.
According to (2) and (5), the size of the secondary current is

$$
\begin{aligned}
I_{c}(Y)= & I_{b} F_{1}\left(Y_{1}\right) \\
& +\frac{K I_{b}}{\sigma \sqrt{2 \pi}} \exp \left[-\frac{\left(\Delta I_{f}+I_{\mathrm{fm}} \sin \alpha\right)^{2}}{2 \sigma^{2}}\right] \\
& +\frac{K_{2} I_{b} I_{\mathrm{fm}} \omega}{\sigma \sqrt{2 \pi}} \exp \left[-\frac{\left(\Delta I_{f}+I_{\mathrm{fm}} \sin \alpha\right)^{2}}{2 \sigma^{2}}\right] \\
& \cdot \cos \alpha .
\end{aligned}
$$

Then the temporal change in the secondary waveform (1) can be represented as a multiple Fourier series:

$$
I_{c}(Y)=\sum_{m=-\infty}^{\infty} C_{m}(Y) e^{j m \alpha},
$$

where the series coefficients are determined by

$$
C_{m}(Y)=\frac{1}{2 \pi} \int_{-\pi}^{\pi} I_{c}(Y) e^{-j m \alpha} d \alpha .
$$

Considering (6), we obtain

$$
\begin{aligned}
& C_{m}(Y)=\frac{1}{2 \pi} \int_{-\pi}^{\pi} I_{b}\left[F_{1}\left(Y_{1}\right)\right. \\
& +\frac{K}{\sigma \sqrt{2 \pi}} \exp \left[-\frac{\left(\Delta I_{f}+I_{\mathrm{fm}} \sin \alpha\right)^{2}}{2 \sigma^{2}}\right] \\
& \left.+\frac{K_{2} I_{\mathrm{fm}} \omega}{\sigma \sqrt{2 \pi}} \exp \left[-\frac{\left(\Delta I_{f}+I_{\mathrm{fm}} \sin \alpha\right)^{2}}{2 \sigma^{2}}\right] \cos \alpha\right] \\
& \quad \times e^{-j m \alpha} d \alpha .
\end{aligned}
$$

If there are not additional external cyclic effects with frequencies that are multiples of the focus scanning frequency $\omega$, the coefficients of the Fourier series for $\alpha=m \omega t$ do not depend on $F_{1}\left(Y_{1}\right)$ due to the stochastic nature of this component:

$$
\begin{aligned}
& C_{m}(Y)=\frac{1}{2 \pi} \\
& \cdot \int_{-\pi}^{\pi} I_{b}\left[\frac{K}{\sigma \sqrt{2 \pi}} \exp \left[-\frac{\left(\Delta I_{f}+I_{\mathrm{fm}} \sin \alpha\right)^{2}}{2 \sigma^{2}}\right]\right. \\
& \left.+\frac{K_{2} I_{\mathrm{fm}} \omega}{\sigma \sqrt{2 \pi}} \exp \left[-\frac{\left(\Delta I_{f}+I_{\mathrm{fm}} \sin \alpha\right)^{2}}{2 \sigma^{2}}\right] \cos \alpha\right] \\
& \quad \times e^{-j m \alpha} d \alpha .
\end{aligned}
$$

The relationships defined by (7) and (9) represent a mathematical model for the formation of the secondary waveform 
in plasma given a periodic component in the electron beam's focus current. The mathematical model makes it possible to detect the components of the waveform from a sensor with frequencies $m \omega$, where $m$ is a whole number.

We will introduce symbols for all of the terms, regardless of $\alpha$ :

$$
\begin{aligned}
& A=\frac{I_{b} K}{\sigma \sqrt{2 \pi}} \exp \left[-\frac{\left(\Delta I_{f}\right)^{2}}{2 \sigma^{2}}\right] \\
& L=-\frac{\Delta I_{f} I_{\mathrm{fm}}}{\sigma^{2}} \\
& P=-\frac{I_{\mathrm{fm}}^{2}}{2 \sigma^{2}} .
\end{aligned}
$$

In trigonometric form, (7) becomes

$$
I_{c}(\alpha)=\frac{d_{0}}{2}+\sum_{m=-\infty}^{\infty}\left[d_{m} \cos (m \alpha)+b_{m} \sin (m \alpha)\right]
$$

where the series coefficients are defined by the following expressions using (10), (11), and (12):

$$
\begin{aligned}
& d_{0}=\frac{A}{\pi} \int_{-\pi}^{\pi} \exp \left[L \sin \alpha+P \sin ^{2} \alpha\right] d \alpha \\
& d_{m}=\frac{A}{\pi} \int_{-\pi}^{\pi} e^{L \sin \alpha+P \sin ^{2} \alpha} \cdot \cos m \alpha d \alpha \\
& d_{m}=\frac{A}{\pi} \int_{-\pi}^{\pi} e^{L \sin \alpha+P \sin ^{2} \alpha} \cdot \sin m \alpha d \alpha .
\end{aligned}
$$

In Figure 2, we present the results of numerical calculations of the magnitudes of the harmonic components of the collector current $b_{m}$ for frequencies that are multiples of the focus current modulation frequency as per (16). The amplitude of the first harmonic $b_{1}$ is small and in a certain range of varying focus is proportional to the size of the focus's deviation from a sharp focus.

Using (14)-(17) when analyzing and building process control systems is hampered by the complexity of the integral expressions in the formulas. Therefore, we present integral expressions (15)-(17) as a Maclaurin series over $x$ :

$$
e^{x}=1+\frac{x}{1 !}+\frac{x^{2}}{2 !}+\cdots+\frac{x^{n}}{n !}
$$

where $x=L \sin \alpha+P \sin ^{2} \alpha$.

With sufficient accuracy for practical application, we can limit ourselves to five terms of the series in view of the fact that (18) converges over the entire numeric axis. Such a limitation is permissible if $|x|<\sigma$ and is typical for the processes being described.

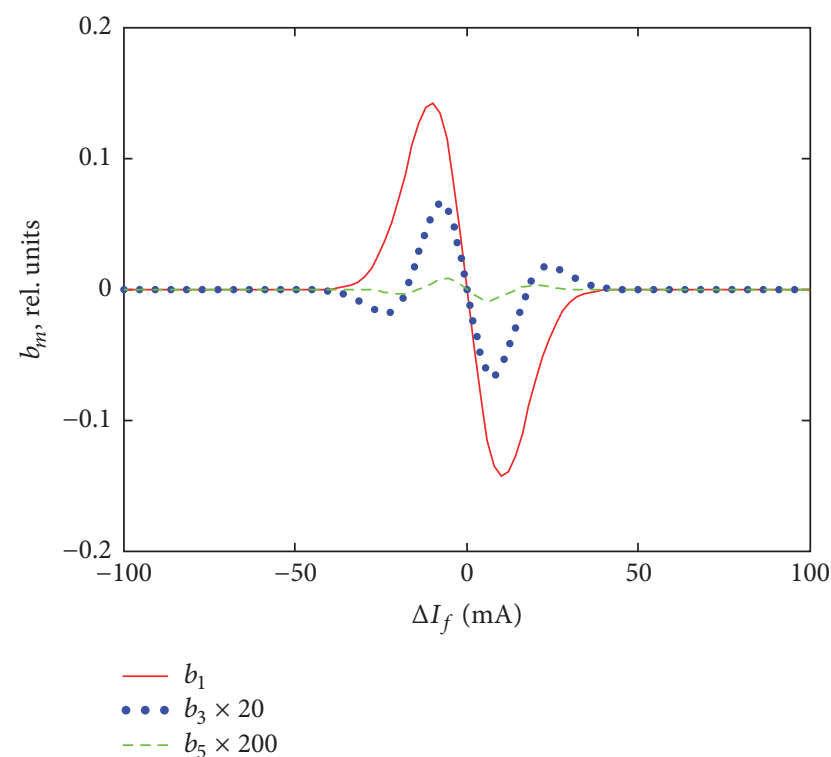

FIGURE 2: Relationship between the focus modes of odd spectral components at frequencies that are multiples of the focus scanning frequency.

Then we have

$$
\begin{aligned}
e^{x}= & +L \sin \alpha+\left(P+\frac{L^{2}}{2}\right) \sin ^{2} \alpha \\
& +\left(L P+\frac{L^{3}}{6}\right) \sin ^{3} \alpha+\left(\frac{P^{2}}{2}+\frac{L P^{2}}{2}\right) \sin ^{4} \alpha \\
& +\frac{L P^{2}}{2} \sin ^{5} \alpha+\frac{P^{3}}{6} \sin ^{6} \alpha .
\end{aligned}
$$

The coefficients of the Fourier series are then computed as

$$
\begin{aligned}
d_{0} & =\frac{A}{\pi} \int_{-\pi}^{\pi} \exp \left[L \sin \alpha+P \sin ^{2} \alpha\right] d \alpha=\frac{A}{2}\left(\frac{5 P^{3}}{24}\right. \\
& \left.+3 P^{2}+L^{2}(1+3 P)+2 P+4\right)=\frac{K}{2 \sqrt{2 \pi} \sigma} \\
& \cdot \exp \left[-\frac{\Delta I_{f}^{2}}{2 \sigma^{2}}\right] \cdot\left[4-\frac{5 I_{\mathrm{fm}}^{6}+288 \Delta I_{f}^{2} I_{\mathrm{fm}}^{4}}{192 \sigma^{6}}\right. \\
& \left.+\frac{4 \Delta I_{f}^{2} I_{\mathrm{fm}}^{2}+3 I_{\mathrm{fm}}^{4}}{4 \sigma^{4}}-\frac{I_{\mathrm{fm}}^{2}}{\sigma^{2}}\right] \\
b_{1} & =\frac{A}{4 \pi} \int_{-\pi}^{\pi} \exp \left[L \sin \alpha+P \sin ^{2} \alpha\right] \sin \alpha d \alpha \\
& =A L\left(\frac{1}{2}+\frac{3 P}{8}+\frac{L^{2}}{16}+\frac{5 P^{2}}{32}\right)=\frac{K I_{b} \Delta I_{f} I_{\mathrm{fm}}}{\sigma^{3} \sqrt{2 \pi}} \\
& \cdot \exp \left[-\frac{\Delta I_{f}^{2}}{2 \sigma^{2}}\right] \cdot\left[\frac{3 I_{\mathrm{fm}}^{2}}{16 \sigma^{2}}-\frac{8 \Delta I_{f}^{2} I_{\mathrm{fm}}^{2}+5 I_{\mathrm{fm}}^{4}}{128 \sigma^{4}}-\frac{1}{2}\right] .
\end{aligned}
$$




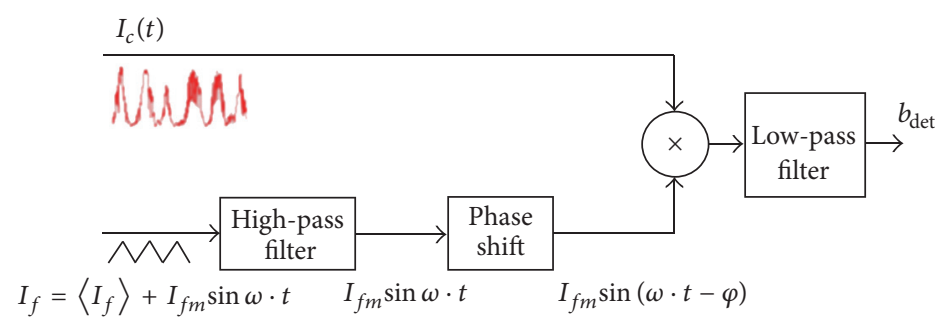

FIGURE 3: Circuit for the synchronous detection method.

Limiting ourselves to three terms for component $d_{1}$, which oscillates with a shift of $\pi / 2$ relative to the focus current's waveform, we obtain

$$
\begin{aligned}
d_{1}= & \frac{A K_{2} I_{\mathrm{fm}} \omega}{K}\left(1+\frac{3 P}{8}+\frac{3 L^{2}}{16}\right) \\
= & \frac{K_{2} I_{\mathrm{fm}} \omega I_{b}}{\sigma \sqrt{2 \pi}} \exp \left[-\frac{\left(\Delta I_{f}\right)^{2}}{2 \sigma^{2}}\right] \\
& \cdot\left[1-\frac{3 I_{\mathrm{fm}}^{2}}{16 \sigma^{2}}\left[1-\frac{\Delta I_{f}^{2}}{\sigma^{2}}\right]\right] .
\end{aligned}
$$

A computational investigation of (15)-(17) showed that the amplitude of the remaining components was one or more orders of magnitude less than the amplitude of the first harmonic, so in our representation of the waveform we limit ourselves to only the first harmonics. Thus, the waveform, represented as a Fourier series, becomes

$$
I_{c}(\alpha)=\frac{d_{0}}{2}+d_{1} \cos \alpha+b_{1} \sin \alpha,
$$

where coefficients $d_{0}$ and $b_{1}$ are defined by (20) and (21). Expressions (20)-(23) are a mathematical model of a sensor for the secondary current in plasma during electron-beam welding with focus scanning.

To evaluate the correctness of the assumptions made to obtain (23) and to verify the adequacy of the resulting model of the formation of the secondary waveform during EBW with electron-beam focus scanning, the modeled results were compared with experimental data. Four factors were varied: beam current, $I_{b}$, focus mode, $\Delta I_{f}$, electron-beam focus scanning frequency, $f=\omega / 2 \pi$, and amplitude of the focus scanning, $I_{\mathrm{fm}}$.

During welding passes, a computerized measurement system with a multichannel analog-digital interface was used to record the secondary current in the plasma. Simultaneously, the waveforms proportional to the current in the deflection coils were recorded. The results of the recording were written to a file for subsequent processing.

The amplitudes of the waveform of the secondary current in plasma at the focus scanning frequency were computed with samples of experimental data using a synchronous detection method [31-33]. The method's algorithm is explained by the circuit in Figure 3. The variable component $I_{\mathrm{fm}} \sin \omega \cdot t$. Then the phase of this component is shifted,

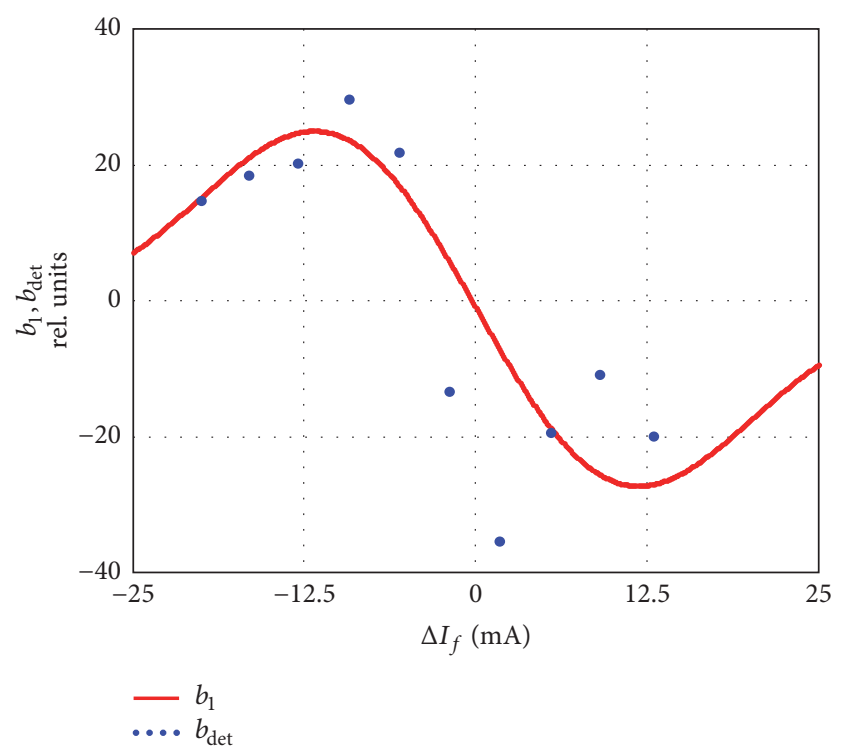

FIgURE 4: Approximation of the amplitude of the first harmonic $b_{1}$ and experimental data points corresponding to values $b_{\text {det }}$. Beam current $I_{b}=31 \mathrm{~mA}$. Focus current modulation amplitude $I_{\mathrm{fm}}=$ $10 \mathrm{~mA}$. Electron-beam scanning focus frequency $f=686 \mathrm{~Hz}$.

if necessary: $I_{\mathrm{fm}} \sin (\omega \cdot t-\varphi)$. The resulting waveform is multiplied by the waveform of the secondary current in plasma, and the result is averaged over time:

$$
b_{\mathrm{det}}=\frac{\int_{0}^{t_{0}} I_{c}(Y) I_{\mathrm{fm}} \sin (\omega t-\varphi) d t}{t_{0}} .
$$

Given zero phase shift and a sufficient waveform sample time $\left(t_{0} \rightarrow \infty\right)$, the result of transformation (24) approaches the value calculated by $(21)\left(b_{\text {det }} \rightarrow b_{1}\right)$. Given $\pi / 2$ phase shift, (22) lets us calculate $d_{1}$. An advantage of the synchronous detection method is its simple implementation in both digital and analog form.

Figures 4 and 5 present curves for amplitudes comprising $b_{1}$, which were calculated using approximation (21), and for experimentally obtained data points for two EBW modes and oscillation parameters. The behavior of the changes in the experimental data confirms the model's results.

A comparative analysis between the model's amplitudes for $b_{1}$ (21) and empirical values (24) confirmed the model's adequacy. The Fisher criterion [34] was 151, the $p$ value was $10^{-10}$, and the correlation coefficient was 0.84 , which indicates 


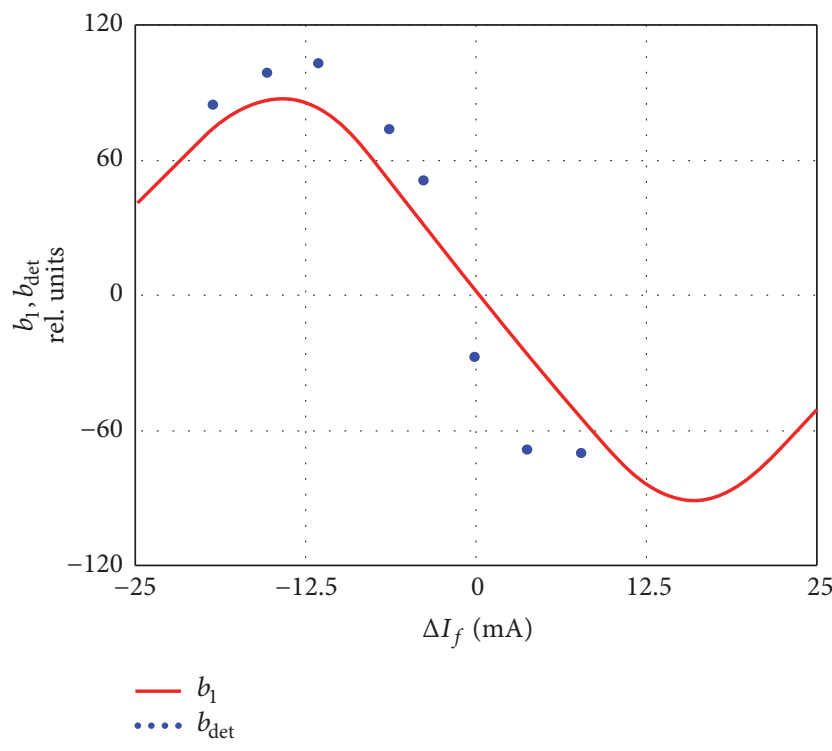

FIGURE 5: Approximation of the amplitude of the first harmonic $b_{1}$ and experimental data points corresponding to values $b_{\text {det }}$. Beam current $I_{b}=40 \mathrm{~mA}$. Focus current modulation amplitude $I_{\mathrm{fm}}=$ $20 \mathrm{~mA}$. Electron-beam scanning focus frequency $f=1400 \mathrm{~Hz}$.

a direct relationship between the resulting function and the observed parameters.

A comparison of experimental data with the modeled results confirms the assumption that given high-frequency focus scanning the behavior of the change in the amplitude of the first harmonic $b_{1}$ was caused by the extreme dependence of the magnitude of the flux density of the energy added by the electron beam on the focus current. It is also significant that a statistical treatment confirmed the modeled results, which indicate the weak influence of the scanning frequency in the range under consideration on the amplitude of the first harmonic.

Figures 6 and 7 present the results of processing the waveform of the secondary current in plasma during EBW with focus spot scanning for underfocused and overfocused modes. For underfocused and overfocused modes, the component of the secondary current in plasma oscillates in phase with the focus scanning waveform, changing signs when moving through sharp focus, but near sharp focus the situation changed drastically (Figure 7).

As predicted by our model, in sharp focus the amplitude of the first harmonic $b_{1}$ of oscillations, which are in phase with the oscillations of the focus current, approached zero. At the same time, $d_{1}$ component appears, which has a phase shift of $\pi / 2$ relative to the focus scanning waveform. The value of this component is virtually unchanged in comparison with $b_{1}$ values in focus conditions far from sharp focus.

Figure 9 presents the results of modeling the formation of the waveform when processing using the synchronous integration method for the three modes of electron-beam focus. The appearance of the resulting curves agrees well with the experimental data (Figures 6, 7, and 8). In underfocused mode, the size of the secondary waveform increases when the focus spot is raised, but in overfocused mode the waveform is maximum when the focal point is lowered in the penetration channel. Given sharp focus, the amplitude of the secondary waveform remains virtually unchanged, but the phase of its oscillations shifts by $\pi / 2$ relative to the scanning waveform.

Despite the fact that in building the model we essentially used an approach accepted for analysis of optimal controllers the results obtained have a number of fundamental differences. In an optimal controller, when moving through an extreme all components at the effect frequency approach zero. In our case, it is not to say that the amplitude of the first harmonic is equal to zero. Its amplitude remains virtually unchanged, but there is a change in phase; and at sharp focus the phase shift is $\pi / 2$. Thus, phase relationships must be used in automatic control. Moreover, the amplitudes of the components oscillating in phase $\left(b_{1}\right)$ or with a shift of $\pi / 2$ (component $d_{1}$ ) can be measured in order to calculate the phase. For example, given sharp focus, the shift in the phase of the oscillations of the secondary current relative to the current of the scanning waveform is $\pi / 2$, which corresponds to zero for $b_{1}$ or a maximum value for $d_{1}$.

When the focus mode is stabilized using (21) the system's stable working range, in the general case, is limited by a certain maximum deviation from sharp focus. The size of this deviation must not go beyond the range of monotonic change in waveform $b_{1}$. For electron guns designed by the Paton Electric Welding Institute, this range is $\Delta I_{f} \in(-15,15) \mathrm{mA}$.

Other shortcomings of the method include the fact that the automatic control system is affected by the arrangement and sizes of the electron collector, the amounts of bias voltage on it, and so forth. All of the relationships were obtained with accuracy to a constant coefficient dependent on the factors listed above. The solution is to directly calculate a magnitude that characterizes the shift in the phase of the first harmonic of the secondary waveform relative to the waveform of the focusing coil current:

$$
\begin{aligned}
& \frac{b_{1}}{d_{1}} \\
& =\frac{C\left(\Delta I_{f} / \omega \sigma^{2}\right)\left[3 I_{\mathrm{fm}}^{2} / 16 \sigma^{2}-\left(8 \Delta I_{f}^{2} I_{\mathrm{fm}}^{2}+5 I_{\mathrm{fm}}^{4}\right) / 128 \sigma^{4}-1 / 2\right]}{\left[1-\left(3 I_{\mathrm{fm}}^{2} / 16 \sigma^{2}\right)\left[1-\Delta I_{f}^{2} / \sigma^{2}\right]\right]},
\end{aligned}
$$

where $C$ is an experimentally determined coefficient that depends on the material being welded (for steel, $C \sim$ $\left.10^{-2} \mathrm{~A} / \mathrm{s}\right)$.

Figure 10 shows the relationship between the tangent of the phase shift of the waveform of the secondary current in plasma relative to the focus scanning waveform $b_{1} / d_{1}$ and the focus mode $\Delta I_{f}$. Clearly, this relationship is linear over the entire range. Additionally, as has already been stated, the transition to phase relationships increases the reproducibility of the results by reducing the influence of the dimensions of the secondary current sensor (electron collector) and its position relative to the welding area.

2.2. Development of an Automatic Focus Control System for Electron-Beam Welding. In the previous section we showed that the shift in the phase of this harmonic relative to the 


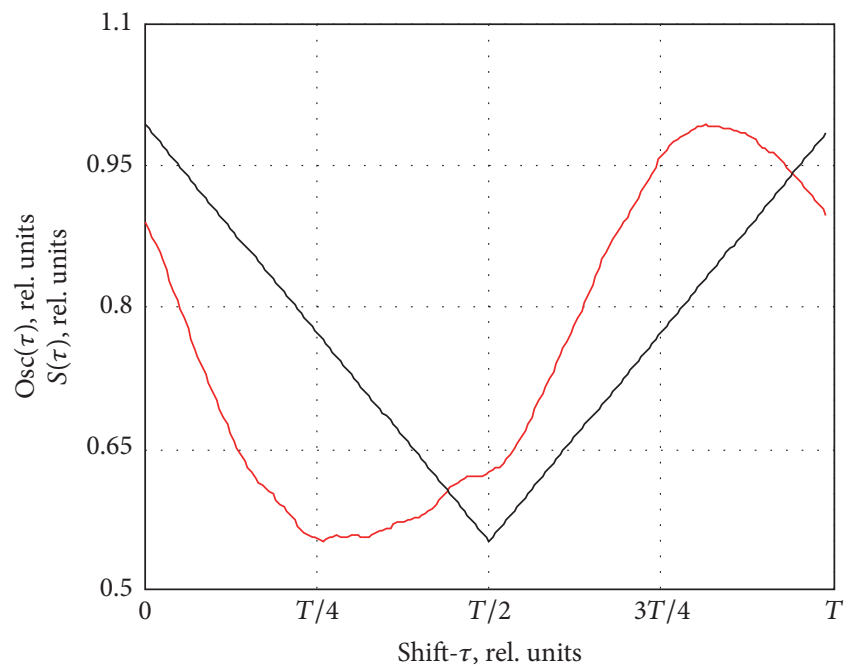

(a)

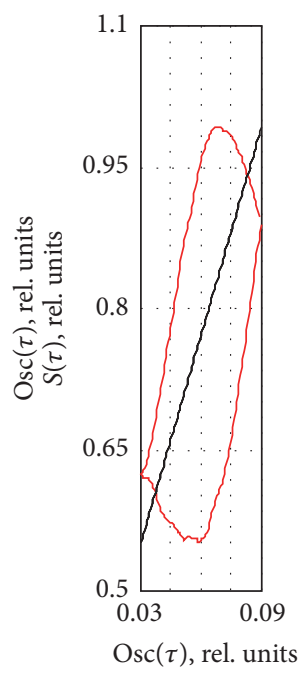

(b)

Figure 6: Results of processing the waveform of the secondary current in plasma during EBW with focus spot scanning $(P=2.5 \mathrm{~kW})$, underfocused $\left(\Delta I_{f}=-15 \mathrm{~mA}\right)$, oscillation frequency $f=966 \mathrm{~Hz}$, and focus current modulation amplitude $I_{\mathrm{fm}}=8 \mathrm{~mA}$ : (a) function $S(t)$, obtained when processing the waveform of the secondary current in plasma using the synchronous accumulation method; (b) $S(t)$ in the phase plane.

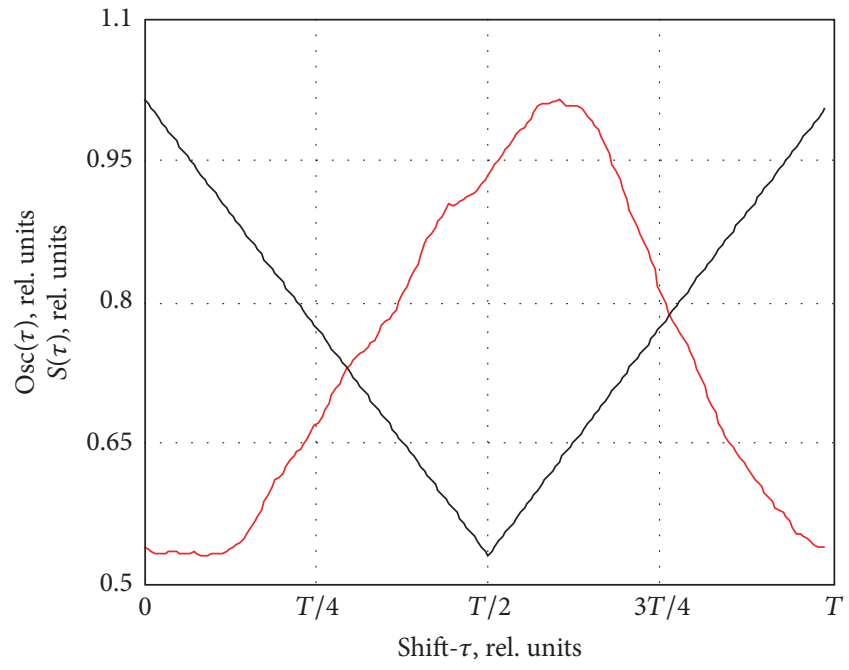

(a)

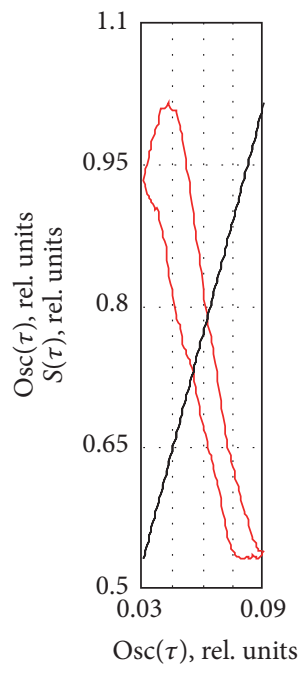

(b)

FIGURE 7: Results of processing the waveform of the secondary current in plasma during EBW with focus spot scanning $(P=2.5 \mathrm{~kW})$, overfocused $\left(\Delta I_{f}=-15 \mathrm{~mA}\right)$, oscillation frequency $f=966 \mathrm{~Hz}$, and focus current modulation amplitude $I_{\mathrm{fm}}=8 \mathrm{~mA}$ : (a) function $S(t)$, obtained when processing the waveform of the secondary current in plasma using the synchronous accumulation method; (b) $S(t)$ in the phase plane.

scanning waveform depends on the focus mode and becomes $\pi / 2$ given the focus current that maximizes the flux density of the energy added by the electron beam. The value that characterizes the phase shift in $b_{1}$ changes in accordance with (29) and becomes zero when the beam is in sharp focus.

Figure 11 presents the functional diagram, which clarifies the essence of the focus control method for EBW. During electron-beam welding, the electron-beam welding apparatus, which contains (1) an electron gun with (2) a focus coil, (3) an amplifier for the focus coil current, and (4) an electron collector to detect the secondary current, uses (5) a modulation block to modulate the electron beam's focus current with frequency $\omega$ and circuitry containing (6) a bias voltage source and (7) load resistor, which are connected in series to the electron collector, to detect the secondary current. The voltage from (7) the load resistor, which is processed with (8) a high-frequency filter with a cutoff frequency of $\omega_{0} \ll \omega$ in order to eliminate the constant component in the detected waveform and is proportional to the size of the secondary current, is supplied to (9) the synchronous detection block. 


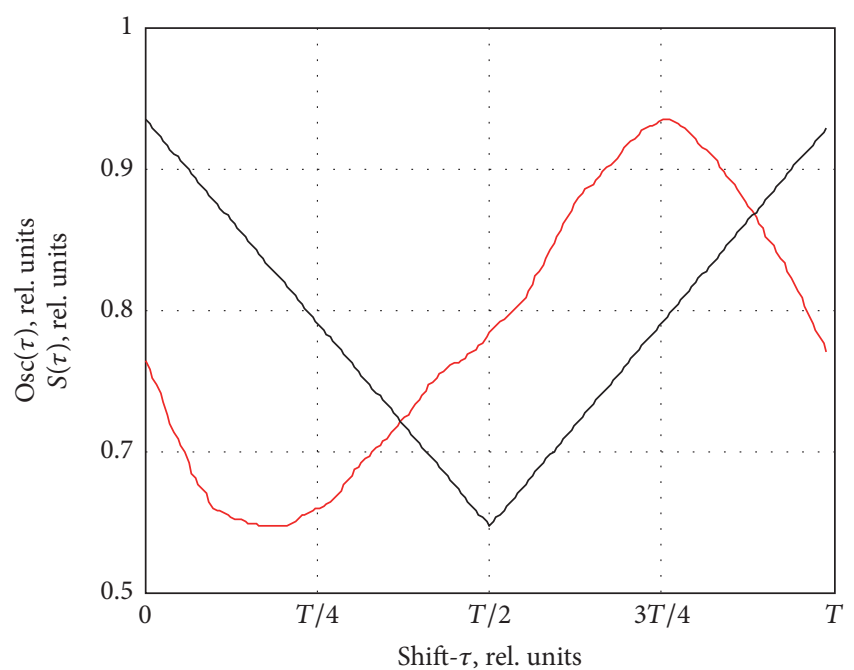

(a)

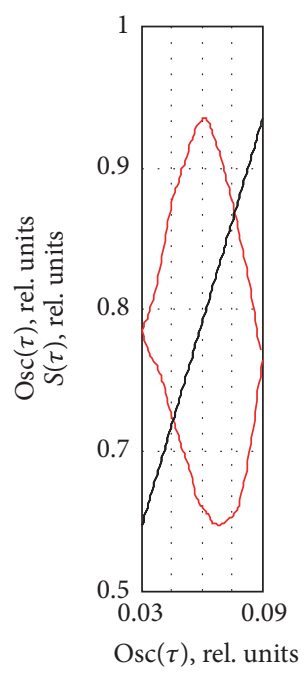

(b)

FIGURE 8: Results of processing the waveform of the secondary current in plasma during EBW with focus spot scanning $(P=2.5 \mathrm{~kW})$, overfocused $\left(\Delta I_{f}=-15 \mathrm{~mA}\right)$, oscillation frequency $f=966 \mathrm{~Hz}$, and focus current modulation amplitude $I_{\mathrm{fm}}=8 \mathrm{~mA}$ : (a) function $S(t)$, obtained when processing the waveform of the secondary current in plasma using the synchronous accumulation method; (b) $S(t)$ in the phase plane.

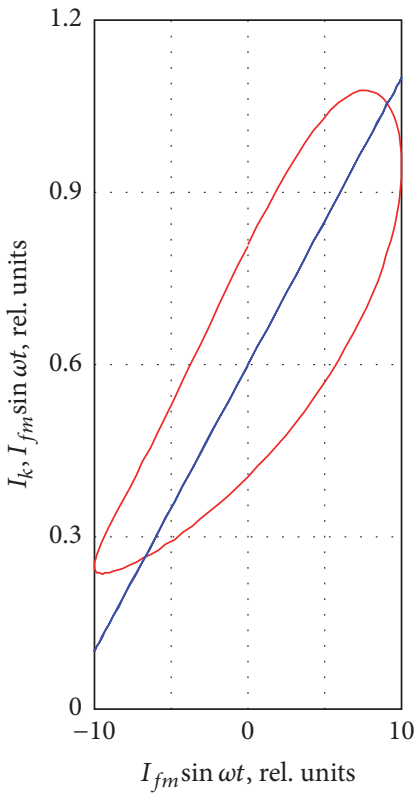

(a)

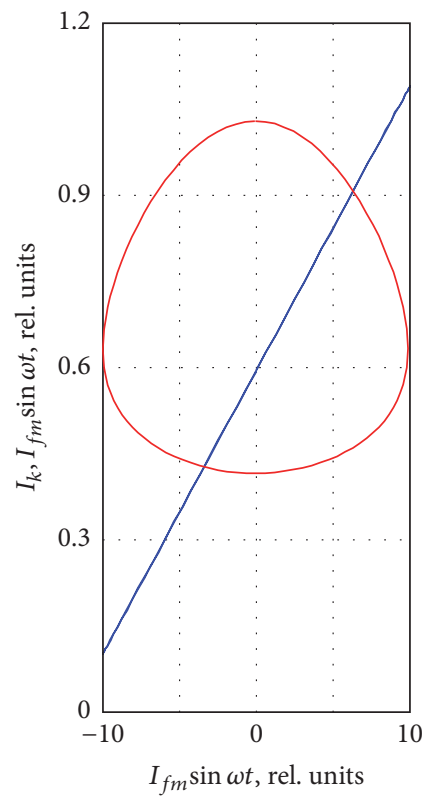

(b)

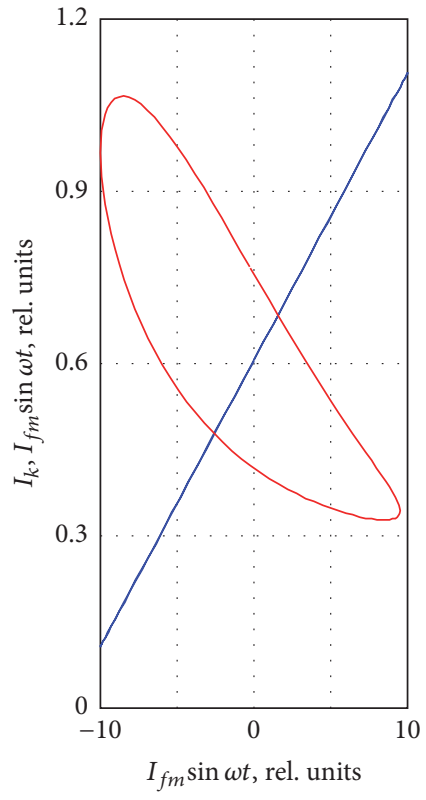

(c)

FIGURE 9: Results of modeling the formation of the waveform of the secondary current in plasma when processing using a synchronous accumulation method during EBW with focus spot scanning: (a) underfocused $\left(\Delta I_{f}=-15 \mathrm{~mA}\right)$; (b) sharp focus $\left(\Delta I_{f}=0 \mathrm{~mA}\right)$; (c) overfocused $\left(\Delta I_{f}=+15 \mathrm{~mA}\right)$.

The (9) synchronous detection block processes the waveform, which is proportional to the size of the secondary current, and the waveform from (5) the modulation block in order to determine the size of the harmonic of the secondary waveform $b_{1}$, which is in phase with the waveform from the modulation block. The amplified waveform is supplied to (10) the control block, where it is compared with the value of $b_{1 \text { est }}$ previously determined by (25), and the control waveform is output to (3) the amplifier for the focus coil current. The preferred shape of (4) the electron collector is a flat disk with an aperture, installed above the welding zone perpendicular to the axis of the electron beam. The recommended distance from the collector to the welding zone is $20-50 \mathrm{~mm}$, which lowers the level of ion-acoustic instability in plasma 


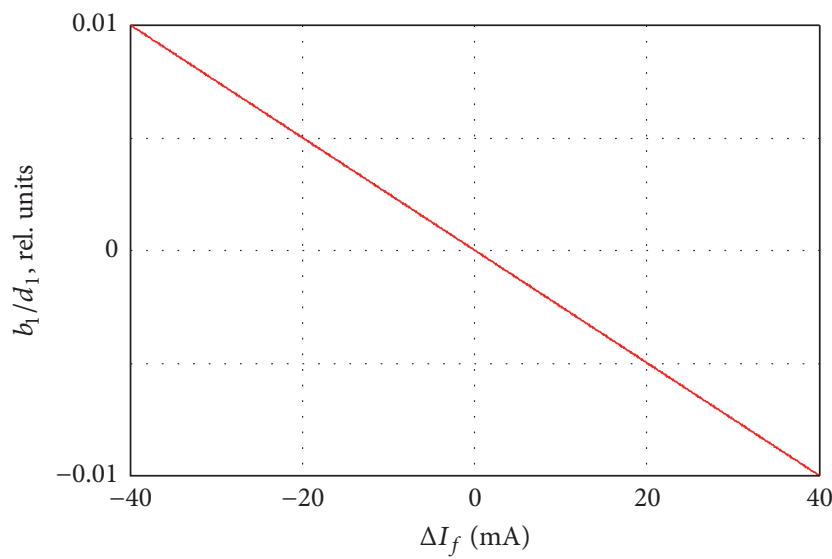

FIGURE 10: Change in the phase shift of the waveform of the secondary current in plasma relative to the scanning focus waveform as a function of focus mode.

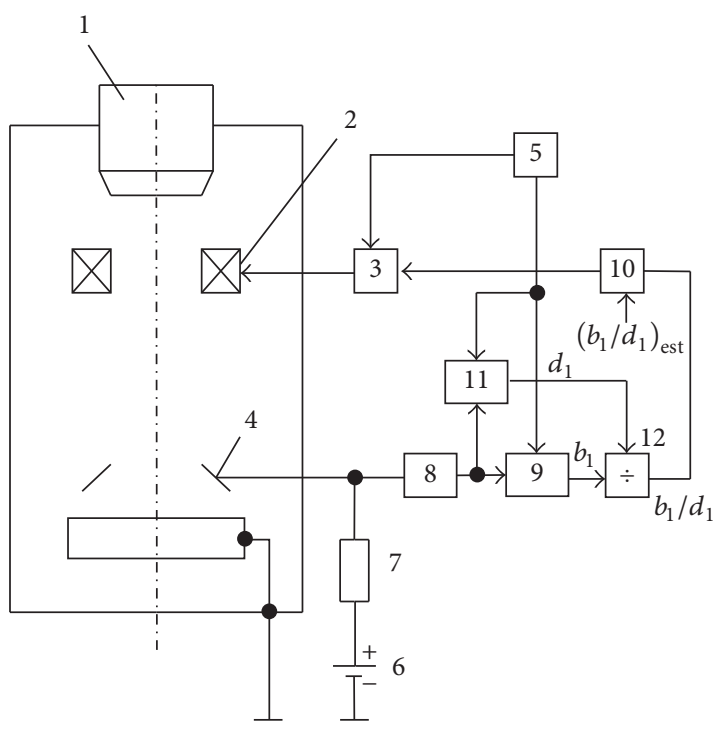

FIGURE 11: Functional diagram of the modified focus control system: 1, electron gun; 2 , focus coil; 3 , amplifier for the focus coil current; 4 , electron collector; 5 , modulation block; 6 , bias voltage source; 7 , load resistor; 8 , high-frequency filter; 9 , synchronous detection block for harmonic $b_{1} ; 10$, control block; 11 , synchronous detection block for harmonic $d_{1} ; 12$, division block.

$[28,34]$, which arises when exciting a non-self-sustained discharge. The synchronous detection block, whose operation is explained by the block diagram in Figure 10, processes the secondary waveform in accordance with (24).

Waveforms from blocks 9 and 11 for the synchronous detection of harmonics $b_{1}$ and $d_{1}$ reach (12) the division block, where the tangent of the shift in the phase of the secondary waveform relative to the scanning waveform $b_{1} / d_{1}$ is calculated. Then $b_{1} / d_{1}$ is used to control the focus with the help of (10) the control block. Moving to phase relationships makes the system more universal and increases the reproducibility of the results by reducing the influence of the position and dimensions of the secondary current sensor (electron collector).

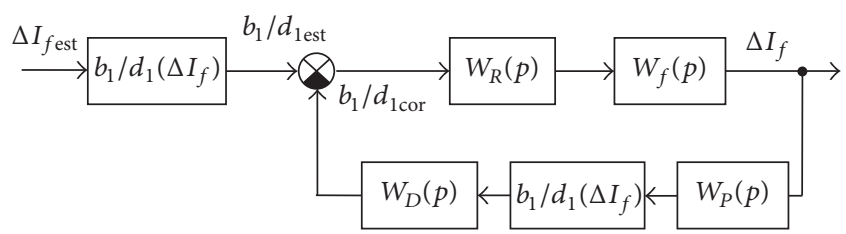

FIGURE 12: Structural diagram of the automatic focus control system.

To calculate the controller parameters and analyze the stability of the control system in stable operation, the operation of the control system was simulated in Matlab Simulink. A structural diagram of the automatic focus control system in stable operation is given in Figure 12.

The specified focus mode $\Delta I_{\text {est }}$ is treated as an input signal. Its current value $I_{f}$ is taken as the output value. The $b_{1} / d_{1}\left(I_{f}\right)$ section corresponds to an equation that describes the link between the focus mode and the shift in the phase of the secondary waveform relative to the scanning waveform. $W_{R}(p)$ is the transfer function for the PID controller

$$
W_{R}(p)=K_{p}\left(1+\frac{1}{T_{I} p}+T_{D} p\right)
$$

where $K_{p}$ is the proportional coefficient, $T_{I}$ is the integration constant, and $T_{D}$ is the differentiation constant.

The focus control system includes an amplifier for the deflection coil current with the coil itself. We provide the circuit's transfer function in the form of a first-order relaxation circuit:

$$
W_{f}(p)=\frac{K_{f}}{T_{f} p+1},
$$

where $T_{f}$ is the characteristic time for the stabilization channel based on the focus current based on a constant value (we took $0.1 \mathrm{~s}$ ) and $K_{f}$ is the channel's transfer coefficient.

$W_{P}(p)$ is the control object's transfer function, which establishes the link between the parameters of the current in plasma during EBW and the focus mode. It is a first-order relaxation circuit:

$$
W_{P}(p)=\frac{K_{Q}}{T_{Q} p+1},
$$

where constant time $T_{\mathrm{Q}}$ represents the behavior of thermal emissivity when changing the power density and $K_{Q}$ is the amplification coefficient. Experimental data [26, 35] indicate that the secondary waveform is weakly related to the focus scanning frequency, which suggests that the secondary waveform's size responds quite rapidly to changes in power density. To estimate the value of $T_{Q}$, we can use the estimated energy accumulation time $\tau \sim 10^{-4}$ from [36].

The focus sensor contains a synchronous detection block (Figure 10). This block's operation assumes that lowfrequency filters with a cutoff frequency of $\omega_{0} \ll \omega$ will be used on the output. We approximate the focus sensor's 


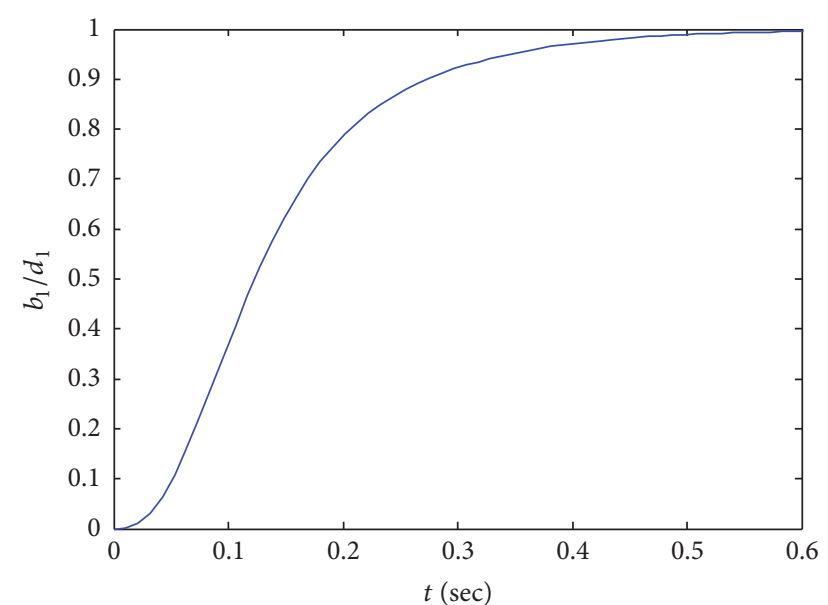

FIgURE 13: Transient process in an open-loop system.

transfer function $W_{D}(p)$ using a characterization of a 2-order Butterworth filter $[37,38]$ :

$$
W_{D}(p)=\frac{1}{T_{L B}^{2} p^{2}+\sqrt{2} T_{f} p+1},
$$

where $T_{L B}=0.033 \mathrm{~s}$ is the filter's time constant (inverse of the cutoff frequency).

The control system was analyzed and synthesized using well-known methods of the theory of continuous systems of equations. The transient process in an open-loop system, which was received during our simulation using Matlab Simulink, is shown in Figure 13. The CHR method (Chien, Hrones, and Reswick) [39, 40] was used to tune the PID controller, which is part of the control system. Unlike the Ziegler-Nichols method, in which a dampening ratio is used as the quality criterion, the CHR method uses the maximum buildup rate in the absence of overshoot or in the presence of no more than $20 \%$ overshoot. Such a criterion makes it possible to achieve a greater stability margin than in the ZieglerNichols method. Coefficients $K_{p}, T_{I}, T_{D}$ of the PID controller's transfer function are, respectively, 2, 0.1, and 0.02 after calculation and tuning.

Logarithmic amplitudinal and phase characteristics of the open loop system, which were created in Matlab Simulink (Figure 14), demonstrate the stability of the system. The phase stability margin is $62.2^{\circ}$, while the amplitude stability margin is $12.6 \mathrm{~dB}$.

Figure 15 presents the transient characteristics in a closedloop system. The control time was $0.315 \mathrm{~s}$ with maximum overshoot of $6 \%$ and a statistical error term of 0 .

A prototype of the focus control system was developed to implement the described control method. A block diagram of the prototype is given in Figure 16. The generator and amplifier create a focus current modulation waveform, which is applied to the adapter module at the constant level of the focus current. In the adapter module and by the sensors installed in the EBW apparatus's processing chamber, measurements are made, the waveform of the secondary current in plasma is synchronously detected, and the size of the shift
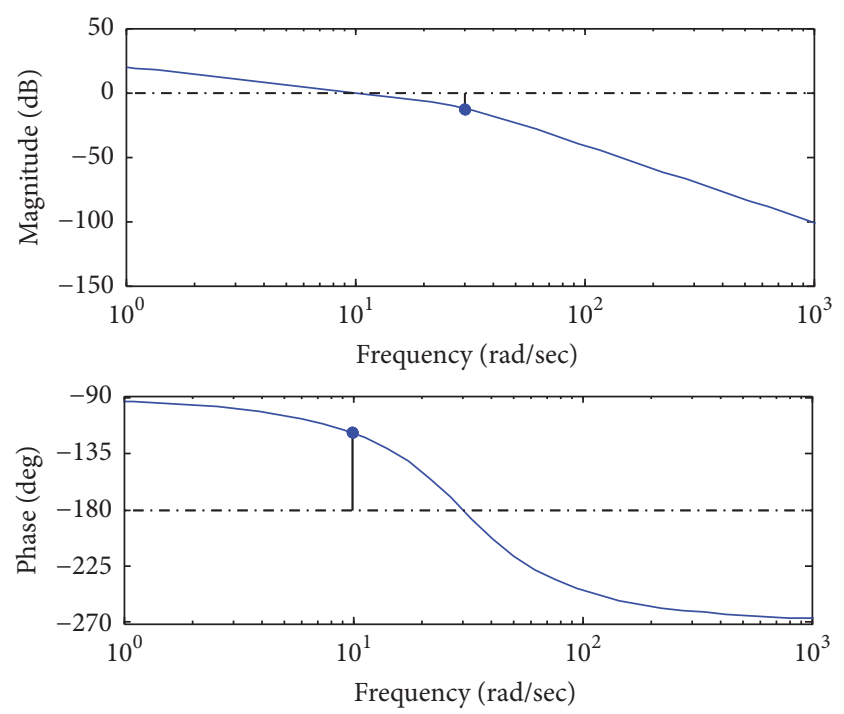

FIGURE 14: Logarithmic amplitudinal and phase characteristics of the open loop system.

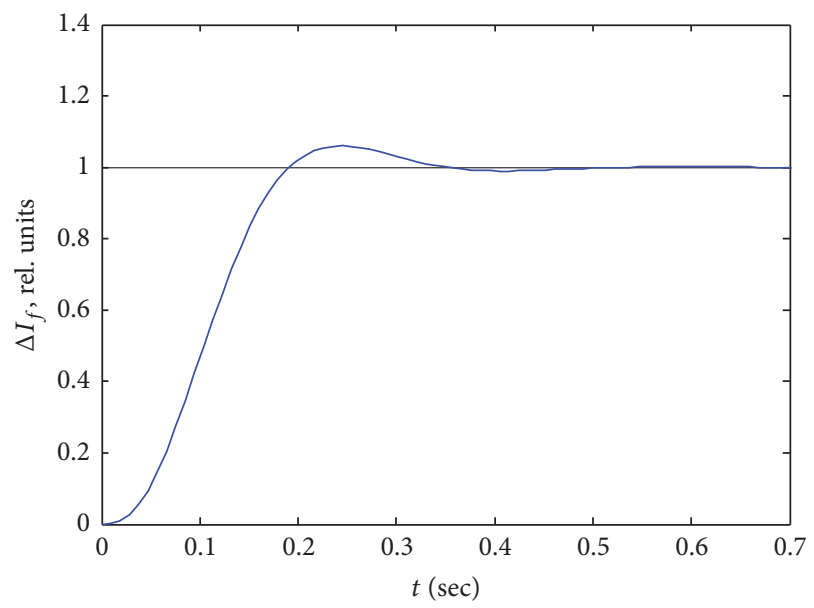

FIGURE 15: Transient process in a closed-loop system.

in its phase relative to the scanning waveform is determined. The value that characterizes the phase shift in analog form reaches the control block, implemented using a Siemens S7-1200 6ES7211-1AE31-0XB0 controller with a 6ES72324HA30-0XB0 analog output board. The control block uses PID control to automatically maintain the focus. Interaction with an operator happens through an industrial computer connected to the control block via an Ethernet network.

The final tuning of the PID controller took place during full-scale testing of the system. The prototype was tested on an ELA-6VCh electron-beam welding apparatus. Parameters are the following: accelerating voltage $60 \mathrm{kV}$; the welding current varied from 40 to $60 \mathrm{~mA}$. The passes were made on specimens made of $12 \mathrm{Kh} 18 \mathrm{~N} 10 \mathrm{~T}$ steel. The electron collector was located at a distance of $40 \mathrm{~mm}$ from the welding zone and was a ring with a surface area of $8 \mathrm{~cm}^{2}$.

Figure 17 demonstrates the prototype's test results. During testing, the influence of external disturbances resulting in 


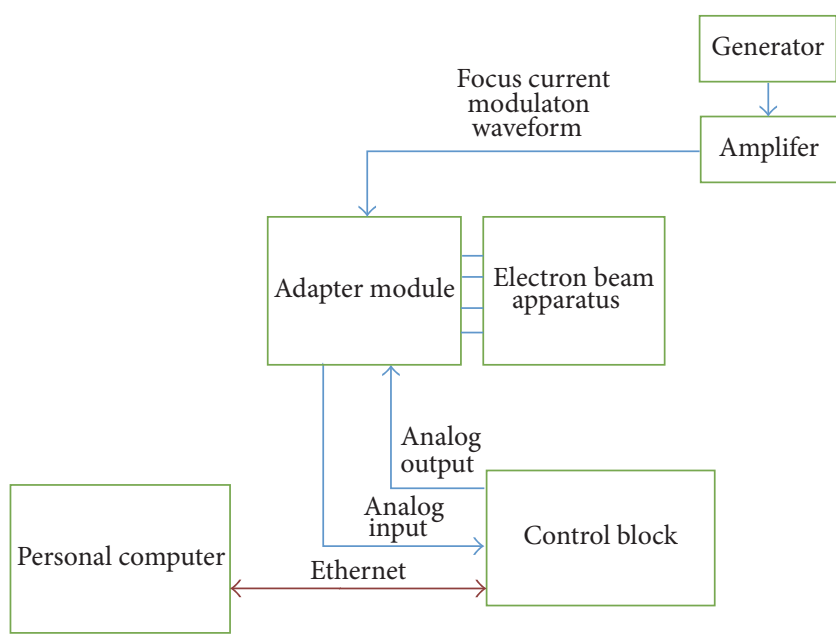

FIGURE 16: Structural diagram of an experimental prototype of the focus control system using the parameters of the secondary current in plasma.

focus drift was modeled by changing the working distances in the welding process (distance between the piece being welded and the electron gun). The system ensures stable focus and, consequently, stabilizes the required geometric parameters in the produced beads.

\section{Conclusions}

(1) We have established that the parameters of the secondary current in plasma, which arises during EBW, carry information about the melting conditions and focus of the electron beam and can be used during process automation.

(2) The model we have obtained describes the mechanism of the formation of the secondary waveform in plasma and changes in its parameters when changing the conditions of EBW with focus spot scanning. We have established that during EBW with focus scanning the first harmonic dominates in the waveform of the secondary current in plasma. Its amplitude remains virtually unchanged, but the phase changes from 0 to $\pi$ when moving from an underfocused state to an overfocused state.

(3) A comparison of modeled results with experimental data confirms the adequacy of the model. The importance of the frequency in the derived relationships is substantial. This result indicates that the extreme nature of the dependence of the current in plasma on the focus current is due to dependence on the electron beam's energy flux density and not only the geometry of the penetration channel. The best results for focus control are obtained when transitioning to phase relationships. We have shown that the value that characterizes the phase shift in the first harmonic of the secondary waveform relative to the focusing coil
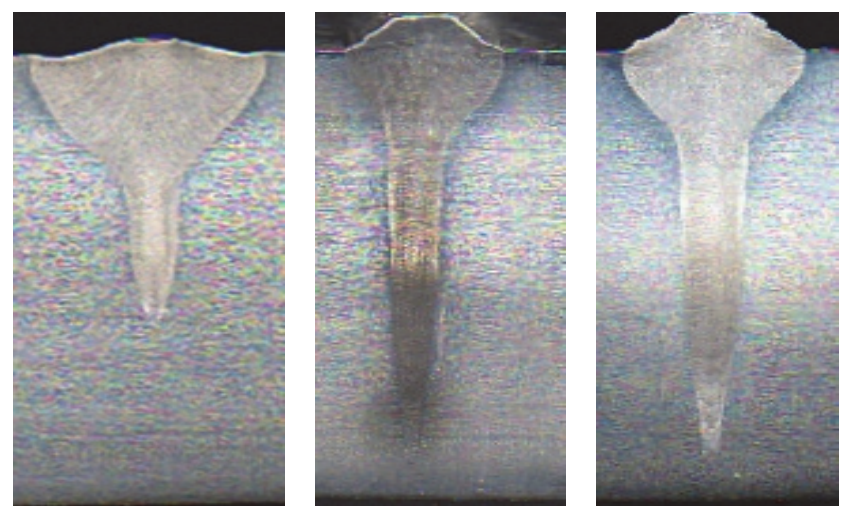

(a) Without focus control
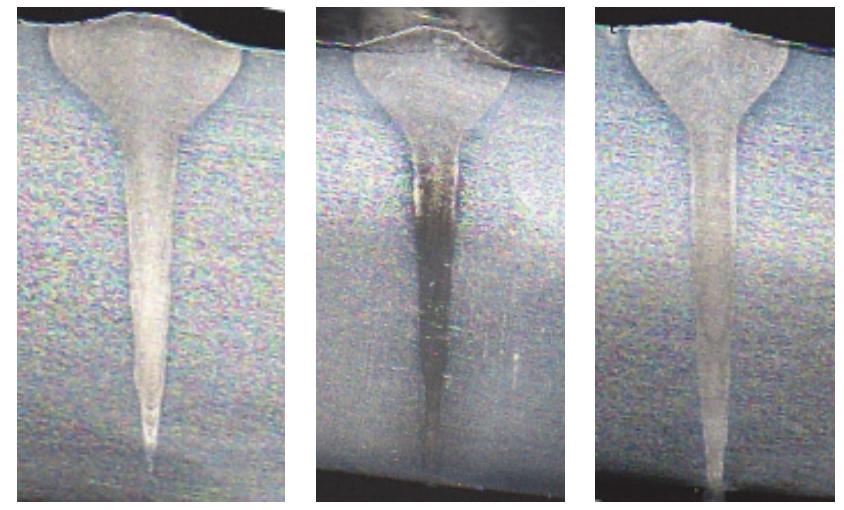

(b) With focus control

FIGURE 17: Cross sections of beads given varying working distances.

current depends monotonically on the focus mode and not on the beam's current.

(4) We outline the principles for building an EBW focus control system based on parameters of the secondary current in plasma. We simulated the operation of the control system's circuits. We studied the stability of the synthesized system and the choice of optimal parameters for the controller in the control system. Use of the system ensures stable focus and, consequently, the achievement of the required geometric parameters in the produced beads, which will result in significant reduction in defective goods during EBW. These measures can reduce the size of allowances in subsequent mechanical processing, which leads to a reduction in the cost of production and improves the energy-efficiency of the welding process. In addition to solving the problems mentioned above, using this operational control and monitoring makes it possible to significantly reduce expenses on the optimization of welding processes, which also makes it possible to reduce the cost of production overall.

\section{Competing Interests}

The authors declare that there is no conflict of interests regarding the publication of this paper. 


\section{Acknowledgments}

This work was supported by a grant from the Perm National Research Polytechnic University.

\section{References}

[1] U. Dilthey, "Estimated determination of kinetics of diffusion phase trans-formations in low-alloy steels in beam welding," in Avtomaticheskaia Svarka, U. Dilthey, A. V. Gumenyuk, and G. A. Turichin, Eds., 2006.

[2] U. Dilthey, A. Goumeniouk, V. Lopota, G. Turichin, and E. Valdaitseva, "Development of a theory for alloying element losses during laser beam welding," Journal of Physics D: Applied Physics, vol. 34, no. 1, pp. 81-86, 2001.

[3] R. Bakish, Introduction to Electron Beam Technology, John Wiley \& Sons, New York, NY, USA, 1985.

[4] G. M. Mladenov, "Physical and thermal processes during electron beam welding," Materials and Manufacturing Processes, vol. 14, no. 3, pp. 331-345, 1999.

[5] P. Petrov, C. Georgiev, and G. Petrov, "Experimental investigation of weld pool formation in electron beam welding," Vacuum, vol. 51, no. 3, pp. 339-343, 1998.

[6] T. DebRoy and S. A. David, "Physical processes in fusion welding," Reviews of Modern Physics, vol. 67, no. 1, pp. 85-112, 1995.

[7] G. Mladenov and S. Sabchevski, "Potential distribution and space-charge neutralization in technological intense electron beams-an overview," Vacuum, vol. 62, no. 2-3, pp. 113-122, 2001.

[8] M. Gabovich, L. Simonenko, I. Soloshenko, and N. Shkorina, "Excitation of ion oscillations in plasma by a fast beam of negative ion," Journal of Experimental and Theoretical Physics, vol. 67, no. 5, pp. 1710-1716, 1975.

[9] U. Dilthey, A. Goumeniouk, O. K. Nazarenko, and K. S. Akopjantz, "Mathematical simulation of the influence of ioncompensation, self-magnetic field and scattering on an electron beam during welding," Vacuum, vol. 62, no. 2-3, pp. 87-96, 2001.

[10] C. Y. Ho, M. Y. Wen, Y. H. Tsai, and C. Ma, "Potential and electron density calculated for freely expanding plasma by an electron beam," Journal of Applied Physics, vol. 110, no. 1, Article ID 013306, 2011.

[11] A. Pinar, B. Wijnen, G. C. Anzalone, T. C. Havens, P. G. Sanders, and J. M. Pearce, "Low-cost open-source voltage and current monitor for gas metal arc weld 3D printing," Journal of Sensors, vol. 2015, Article ID 876714, 8 pages, 2015.

[12] D. Trushnikov, V. Belenkiy, V. Shchavlev, A. Piskunov, A. Abdullin, and G. Mladenov, "Plasma charge current for controlling and monitoring electron beam welding with beam oscillation," Sensors (Basel, Switzerland), vol. 12, no. 12, pp. 17433-17445, 2012.

[13] W. Wang, S. Yamane, T. Koike et al., "Image processing method for automatic tracking of the weld line in plasma robotic welding," The International Journal of Advanced Manufacturing Technology, vol. 86, no. 5, pp. 1865-1872, 2016.

[14] W. Wang, S. Yamane, H. Suzuki et al., "Tracking and height control in plasma robotic welding using digital CCD camera," The International Journal of Advanced Manufacturing Technology, vol. 87, no. 1, pp. 531-542, 2016.

[15] X. Gao, D. You, and S. Katayama, "The high frequency characteristics of laser reflection and visible light during solid state disk laser welding," Laser Physics Letters, vol. 12, no. 7, Article ID 076003, 2015.

[16] D. Y. You, X. D. Gao, and S. Katayama, "Review of laser welding monitoring," Science and Technology of Welding and Joining, vol. 19, no. 3, pp. 181-201, 2014

[17] V. D. Laptenok, A. V. Murygin, and D. V. Tikhonenko, "X-ray sensor for guiding the electron beam on the joint in electronbeam welding," Welding International, vol. 20, pp. 894-900, 2006.

[18] V. Y. Braverman, D. A. Skurikhin, S. G. Bayakin, V. F. Shabanov, and V. V. Bashenko, "Device for focusing and fusion depth controlling by characteristic X-ray during electron beam welding with modulation of focusing level," Svarochnoe Proizvodstvo, vol. 1, pp. 16-19, 1997.

[19] U. Dilhey, "Diagnosis and beam measurement in non-vacuum electron beam welding," Elektrotechnika i Elektronika, vol. 41, no. 5-6, pp. 61-65, 2006.

[20] J. W. Elmer and A. T. Teruya, Enhanced modified faraday cup for determi-nation of power density distribution of electron beams, US, Pat. 6300755, 2001.

[21] J. W. Elmer and A. T. Teruya, "Fast method for measuring power density distribution of non-circular and irregular electron beams," Science and Technology of Welding and Joining, vol. 3, no. 2, pp. 51-58, 1998.

[22] E. Koleva and G. Mladenov, "Signal formation analysis of the electron beam current distribution measurements," Vacuum, vol. 77, no. 4, pp. 457-462, 2005.

[23] J. W. Elmer, A. T. Teruya, and D. W. O’Brien, “Tomographic imaging of noncircular and irregular electron beam current density distributions," Welding Journal, vol. 72, no. 11, p. 493, 1993.

[24] V. Y. Braverman, D. A. Skurikhin, S. G. Bayakin, V. F. Shabanov, and V. V. Bashenko, "Device for focusing and fusion depth controlling by characteristic $\mathrm{x}$-ray during electron beam welding with modulation of focusing level," Svarochnoe Proizvodstvo, vol. 1, pp. 16-19, 1997.

[25] K. Olszewska and K. Friedel, "Control of the electron beam active zone position in electron beam welding processes," Vacuum, vol. 74, no. 1, pp. 29-43, 2004.

[26] D. N. Trushnikov, E. G. Koleva, G. M. Mladenov, V. Y. Belenkiy, and E. S. Salomatova, "Weld formation control at electron beam welding with focal spot scanning," Middle-East Journal of Scientific Research, vol. 16, no. 8, pp. 1062-1068, 2013.

[27] D. N. Trushnikov, V. Y. Belenki'y, G. M. Mladenov, and N. S. Portnov, "Secondary-Emission signal for weld formation monitoring and control in eletron beam welding (EBW)," Materialwissenschaft und Werkstofftechnik, vol. 43, no. 10, pp. 892-897, 2012

[28] D. N. Trushnikov, V. E. Shchavlev, G. M. Mladenov, and L. N. Krotov, "Investigation of processes in the keyhole of electronbeam welding by monitoring the secondary current signal in the plasma," in In-Situ Studies with Photons, Neutrons and Electrons Scattering II, pp. 217-230, Springer International, Berlin, Germany, 2014.

[29] D. N. Trushnikov and G. M. Mladenov, "Numerical model of the plasma formation at electron beam welding," Journal of Applied Physics, vol. 117, no. 1, Article ID 013301, 2015.

[30] V. M. Yazovskikh, D. N. Trushnikov, V. Y. Belen'kii, and L. N. Krotov, "The mechanism of secondary emission processes in electron beam welding with the modulation of the electron beam," Welding International, vol. 18, no. 9, pp. 724-729, 2004. 
[31] E. G. Koleva, G. M. Mladenov, D. N. Trushnikov, and V. Y. Belenkiy, "Signal emitted from plasma during electron-beam welding with deflection oscillations of the beam," Journal of Materials Processing Technology, vol. 214, no. 9, pp. 1812-1819, 2014.

[32] D. N. Trushnikov and V. Y. Belen'kii, "Investigation of the formation of the secondary current signal in plasma in electron beam welding with oscillations of the electron beam," Welding International, vol. 27, no. 11, pp. 877-880, 2013.

[33] J. Max, Méthodes et Techniques de Traitement du Signal et Applications aux Mesures Physiques, vol. 1, Jean-Louis Lacoume, Paris, France, 3rd edition, 1981.

[34] D. N. Trushnikov, G. M. Mladenov, V. Y. Belenkiy, E. G. Koleva, and S. V. Varushkin, "Current-driven ion-acoustic and potential-relaxation instabilities excited in plasma plume during electron beam welding," AIP Advances, vol. 4, no. 4, Article ID 047105, 2014.

[35] D. N. Trushnikov, E. G. Koleva, G. M. Mladenov, and A. V. Shcherbakov, "Weld formation control at electron beam welding with beam oscillations," Bulletin of the Siberian State Aerospace University, vol. 3, no. 55, pp. 224-230, 2014.

[36] I. Y. Smurov, A. A. Uglov, A. M. Lashyn, P. Matteazzi, L. Covelli, and V. Tagliaferri, "Modelling of pulse-periodic energy flow action on metallic materials," International Journal of Heat and Mass Transfer, vol. 34, no. 4-5, pp. 961-971, 1991.

[37] M. Krstic, I. Kanellakopoulos, and P. V. Kokotovic, Nonlinear and Adaptive Control Design, John Wiley \& Sons, New York, NY, USA, 1995.

[38] K. J. Astrom and B. Wittenmark, Adaptive Control, AddisonWesley, Boston, Mass, USA, 2nd edition, 1994.

[39] A. Arora, Y. V. Hote, and M. Rastogi, "Design of PID controller for unstable system," Communications in Computer and Information Science, vol. 140, pp. 19-26, 2011.

[40] J. G. Ziegler and N. B. Nichols, "Optimum settings for automatic controllers," Transactions of the ASME, vol. 64, pp. 759-768, 1942. 


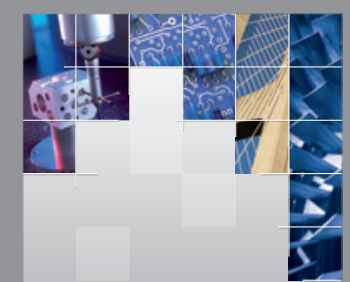

\section{Enfincering}
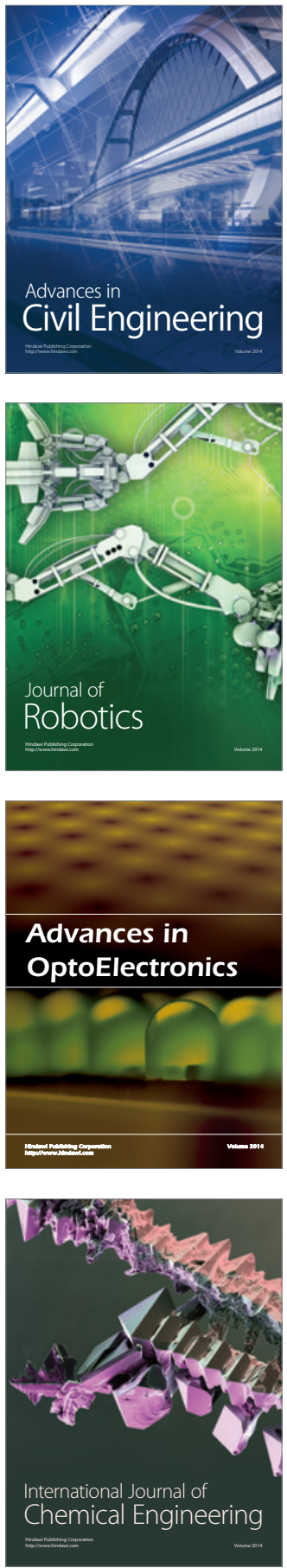

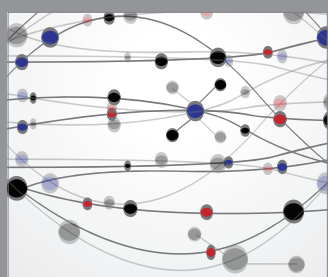

The Scientific World Journal

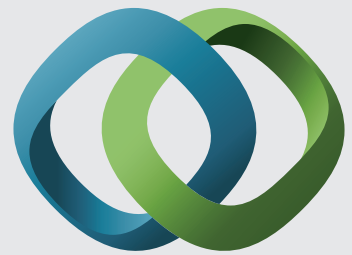

\section{Hindawi}

Submit your manuscripts at

http://www.hindawi.com
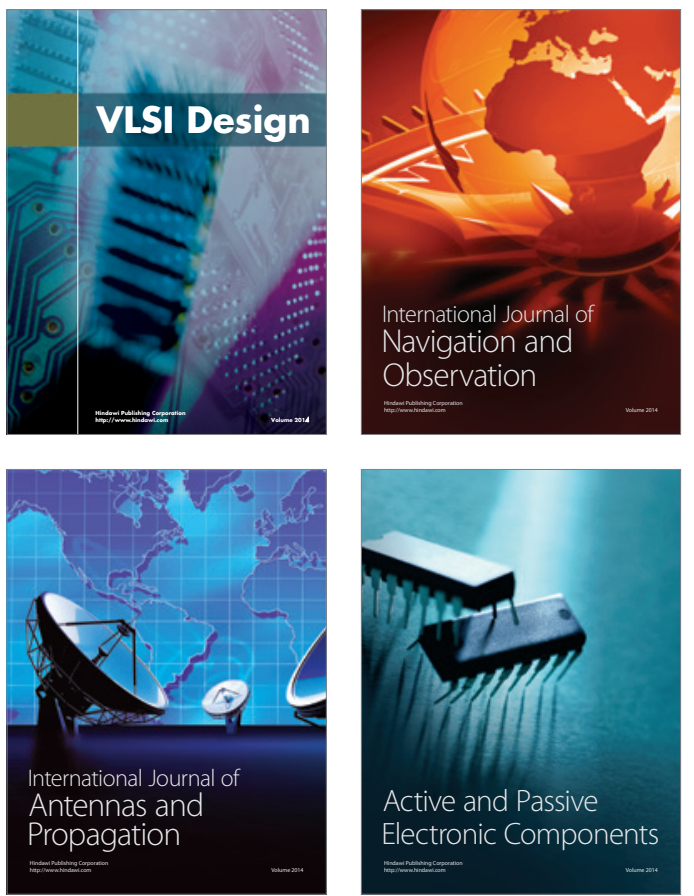
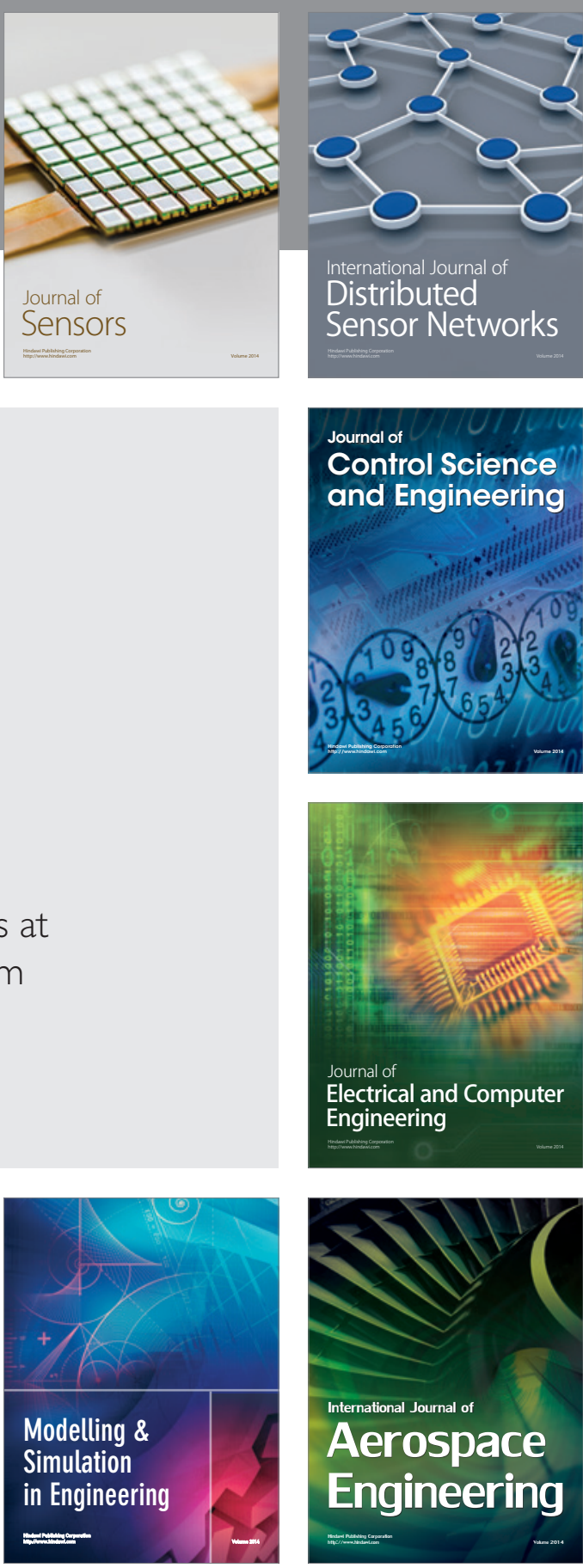

International Journal of

Distributed

Sensor Networks

Journal of

Control Science

and Engineering
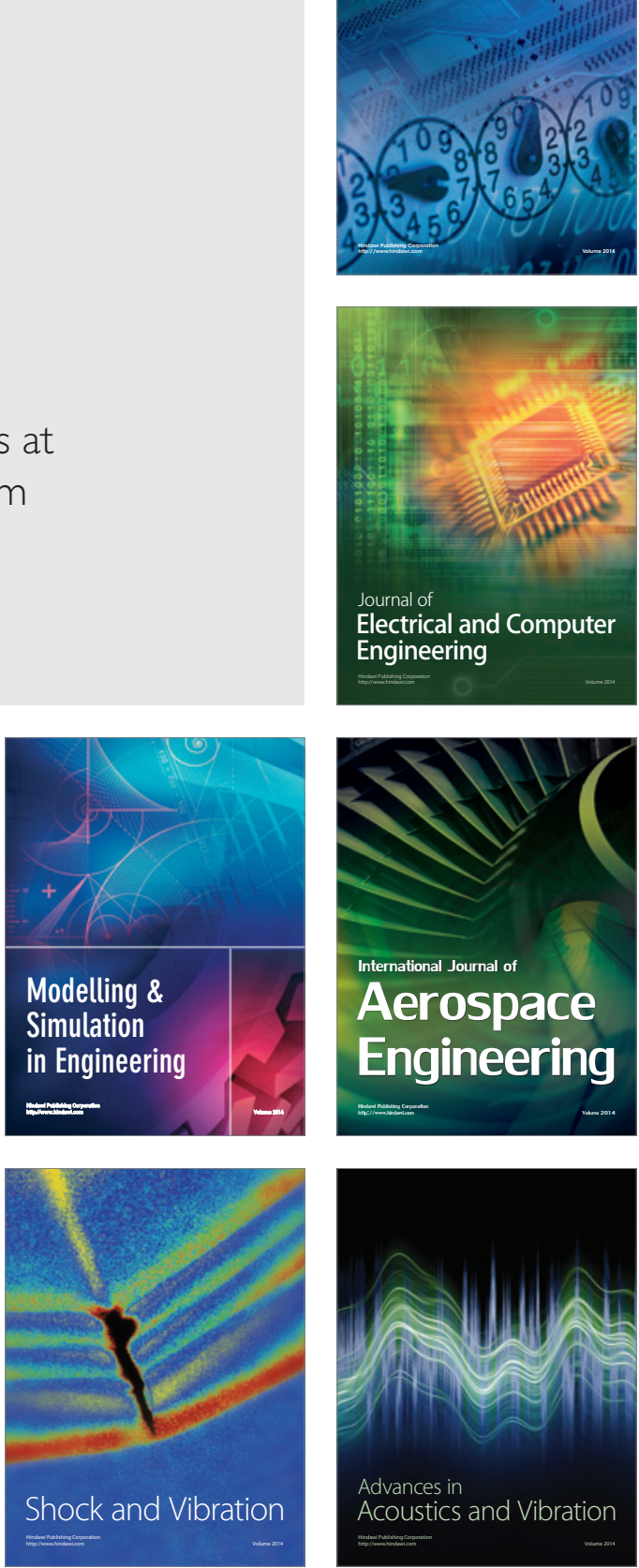